\title{
Minimization of length and curvature on planar curves
}

\author{
Ugo Boscain, Grégoire Charlot and Francesco Rossi
}

\begin{abstract}
In this paper we consider the problem of reconstructing a curve that is partially hidden or corrupted by minimizing the functional $\int \sqrt{1+K^{2}} d s$, depending both on length and curvature $K$. We fix starting and ending points as well as initial and final directions.

For this functional we discuss the problem of existence of minimizers on various functional spaces. We find non-existence of minimizers in cases in which initial and final directions are considered with orientation. In this case, minimizing sequences of trajectories can converge to curves with angles.

We instead prove existence of minimizers for the "timereparameterized" functional $\int\|\dot{\gamma}(t)\| \sqrt{1+K_{\gamma}^{2}} d t$ for all boundary conditions if initial and final directions are considered regardless to orientation.
\end{abstract}

Keywords: geometry of vision, elastica functional, existence of minimizers

AMS classification: 74G65, 74K10, 49J15, 53A04

\section{PRoblems STATEMENTS AND MAIn RESUlts}

Consider a smooth function $\gamma_{0}:[a, b] \cup[c, d] \rightarrow \mathbb{R}^{2}$ (with $a<b<c<d$ ) representing a curve that is partially hidden or deleted in $(b, c)$. We want to find a curve $\gamma:[b, c] \rightarrow \mathbb{R}^{2}$ that completes $\gamma_{0}$ in the deleted part and that minimizes a cost depending both on length $\mathscr{L}(\gamma)$ and curvature $K_{\gamma}$.

The fact that $\gamma$ completes $\gamma_{0}$ means that $\gamma(b)=\gamma_{0}(b)$, $\gamma(c)=\gamma_{0}(c)$. It is also reasonable to require that the directions of tangent vectors (with orientation) coincide, i.e. $\dot{\gamma}(b) \sim \dot{\gamma}_{0}(b), \dot{\gamma}(c) \sim \dot{\gamma}_{0}(c)$ where

$$
v_{1} \sim v_{2} \text { if it exists } \alpha \in \mathbb{R}^{+} \text {such that } v_{1}=\alpha v_{2} .
$$

We call these conditions boundary conditions with orientation. Throughout this paper we assume that starting and ending points never coincide, i.e. $\gamma_{0}(b) \neq \gamma_{0}(c)$, and that initial and final directions are nonvanishing.

In the literature this problem has been deeply studied for its application to problems of segmentation of images (see e.g. [2], [7], [12], [13]) and for the construction of spiral splines [9]. The cost studied in [7], [9], [12] is the total squared curvature $E_{1}[\gamma]=\int_{0}^{\mathscr{L}(\gamma)}\left|K_{\gamma}(s)\right|^{2} d s$ where $s$ is the arclength. The cost studied in [2] is $E_{2}[\gamma]=$

U. Boscain is with CNRS CMAP, École Polytechnique, Palaiseau, France and SISSA, Trieste, Italy boscainesissa.it

G. Charlot is with Institut Fourier - UMR5582, St Martin d'Heres, France Gregoire.Charloteujf-grenoble.fr

F. Rossi is with SISSA, Trieste, Italy and BCAM, Bilbao, Spain rossifresissa.it
$\int_{0}^{\mathscr{L}(\gamma)}\left(1+\left|K_{\gamma}(s)\right|^{2}\right) d s$, while in [13] it is $E_{3}[\gamma]=$ $\int_{0}^{\mathscr{L}(\gamma)}\left(\eta+\left|K_{\gamma}(s)\right|^{2}\right) d s$ with $\eta \rightarrow 0$. Depending on the cost, minimizers may present angles.

In this paper we study the following cost:

$$
J[\gamma]=\int_{b}^{c} \sqrt{\|\dot{\gamma}(t)\|^{2}+\|\dot{\gamma}(t)\|^{2} K_{\gamma}^{2}(t)} d t .
$$

It is an extension of $E_{2}[\gamma]$, see Remark 2 below. Using this cost one can study the existence of minimizers with angles without involving sophisticated functional spaces. Moreover, this cost naturally arises in problems of geometry of vision [8], [16], [17]. This problem has also been studied in [5], where it is defined on the sphere $S^{2}$ instead of $\mathbb{R}^{2}$.

Remark 1: The cost $J$ is invariant both by rototranslation and reparametrization of the curve. Notice also that the minimization of the cost $J_{\beta}[\gamma]:=\int_{b}^{c}\|\dot{\gamma}(t)\| \sqrt{1+\beta^{2} K_{\gamma}^{2}(t)} d t$ with a fixed $\beta \neq 0$ is equivalent to the minimization of $J$ with a suitable change of boundary conditions.

The first question we address in this paper is the choice of a set of smooth curves on which this cost is well-defined. We want $\dot{\gamma}(t)$ and $K_{\gamma}(t)=\frac{\dot{x} \ddot{y}-\dot{y} \ddot{x}}{\left(\dot{x}^{2}+\dot{y}^{2}\right)^{\frac{3}{2}}}$ well-defined, thus it is reasonable to look for minimizers in

$$
\begin{aligned}
\mathscr{D}_{1}:= & \left\{\gamma \in C^{2}\left([b, c], \mathbb{R}^{2}\right) \text { s.t. } \dot{\gamma}(t) \neq 0 \forall t \in[b, c],\right. \\
& \left.\gamma(b)=\gamma_{0}(b), \gamma(c)=\gamma_{0}(c), \dot{\gamma}(b) \sim \dot{\gamma}_{0}(b), \dot{\gamma}(c) \sim \dot{\gamma}_{0}(c)\right\} .
\end{aligned}
$$

Moreover, $\dot{\gamma}(b)$ and $\dot{\gamma}(c)$ are well-defined in this case.

Under this assumption, one of the main results of the paper is the nonexistence of minimizers for $J$.

Proposition 1: There exist boundary conditions $\gamma_{0}(b), \gamma_{0}(c) \in \mathbb{R}^{2}$ with $\gamma_{0}(b) \neq \gamma_{0}(c)$ and $\dot{\gamma}_{0}(b), \quad \dot{\gamma}_{0}(c) \in \mathbb{R}^{2} \backslash\{0\}$ such that the cost (2) does not admit a minimum over the set $\mathscr{D}_{1}$.

To get existence of minimizers one can choose to enlarge the set of admissible curves. Here we choose

$$
\begin{aligned}
\mathscr{D}_{2}:= & \left\{\gamma \in C^{2}([b, c]) \text { s.t. } \sqrt{\|\dot{\gamma}(t)\|^{2}\left(1+K_{\gamma}^{2}(t)\right)} \in L^{1}([b, c]),\right. \\
& \left.\gamma(b)=\gamma_{0}(b), \gamma(c)=\gamma_{0}(c), \dot{\gamma}(b) \sim \dot{\gamma}_{0}(b), \dot{\gamma}(c) \sim \dot{\gamma}_{0}(c)\right\},
\end{aligned}
$$

on which the cost $J[\gamma]$ is defined and always finite.

Remark 2: The cost $J[\gamma]$ on the set $\mathscr{D}_{1}$ coincides with the cost $E_{2}[\gamma]$. Instead, $E_{2}[\gamma]$ is not well defined on $\mathscr{D}_{2}$, since it is not possible in general to perform an arclength parametrization. Then $J[\gamma]$ is an extension of $E_{2}[\gamma]$.

Also on $\mathscr{D}_{2}$ we have non-existence of minimizers for $J$. 
Proposition 2: There exist boundary conditions $\gamma_{0}(b), \gamma_{0}(c) \in \mathbb{R}^{2}$ with $\gamma_{0}(b) \neq \gamma_{0}(c)$ and $\dot{\gamma}_{0}(b), \quad \dot{\gamma}_{0}(c) \in \mathbb{R}^{2} \backslash\{0\}$ such that the cost (2) does not admit a minimum over the set $\mathscr{D}_{2}$.

The basic problem is that we can have a sequence of minimizing curves converging to a non admissible curve. In particular, we can have that each curve $\gamma_{n}$ satisfies given boundary conditions with orientation but the limit curve $\bar{\gamma}$ doesn't satisfy them. See Figure 1.

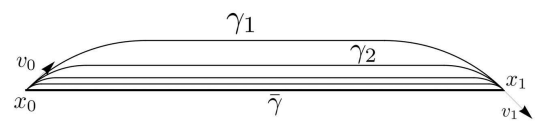

Fig. 1. Minimizing sequence converging to a non-admissible curve (angles at the beginning/end).

The main result of the paper is the existence of minimizers for the cost (2) taking again curves for which $\sqrt{\|\dot{\gamma}(t)\|^{2}\left(1+K_{\gamma}^{2}(t)\right)}$ is integrable, but changing boundary conditions. We only impose conditions on the direction of $\dot{\gamma}$ regardless of its orientation.

As before, fix a starting point $x_{0}$ with a direction $v_{0}$ and an ending point $x_{1}$ with a direction $v_{1}$. Consider planar curves satisfying the following boundary conditions:

$$
\gamma(0)=x_{0}, \dot{\gamma}(0) \approx v_{0}, \gamma(T)=x_{1}, \dot{\gamma}(T) \approx v_{1},
$$

where the identification rule $\approx$ is

$v_{1} \approx v_{2}$ if there exists $\alpha \in \mathbb{R} \backslash\{0\}$ such that $v_{1}=\alpha v_{2}$.

We call them projective boundary conditions. As already stated, we have the following existence result:

Proposition 3: For all boundary conditions $x_{0}, x_{1} \in \mathbb{R}^{2}$ with $x_{0} \neq x_{1}, v_{0}, v_{1} \in \mathbb{R}^{2} \backslash\{0\}$, the cost (2) has a minimizer over the set

$$
\begin{aligned}
\mathscr{D}_{3}:= & \left\{\gamma \in C^{2}([b, c]) \text { s.t. } \sqrt{\|\dot{\gamma}(t)\|^{2}\left(1+K_{\gamma}^{2}(t)\right)} \in L^{1}([b, c]),\right. \\
& \left.\gamma(b)=\gamma_{0}(b), \gamma(c)=\gamma_{0}(c), \dot{\gamma}(b) \approx \dot{\gamma}_{0}(b), \dot{\gamma}(c) \approx \dot{\gamma}_{0}(c)\right\} .
\end{aligned}
$$

Notice that we can have minimizers with cusps, see Fig. 2.

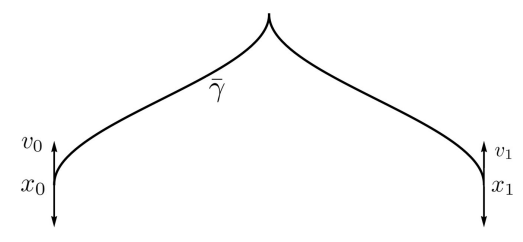

Fig. 2. A minimizer with a cusp.

All the previous results are obtained as consequences of the study of two similar mechanical problems. For problems with boundary conditions with orientation, we consider a car on the plane that can move only forwards and rotate on itself (it is the Dubins' car, see [10]). Fix two points $\left(x_{0}, y_{0}\right),\left(x_{1}, y_{1}\right)$ and two angles $\theta_{0}, \theta_{1}$ in these points measured with respect to the positive $x$-semiaxis. Consider all trajectories $q($.$) steering the car from the point \left(x_{0}, y_{0}\right)$ with the angle $\theta_{0}$ to the point $\left(x_{1}, y_{1}\right)$ with the angle $\theta_{1}$. Our goal is to find the cheapest trajectory with respect to a cost depending both on the length of displacement on the plane and on the angle of rotation on itself. The dynamics can be written as the following control system on the group of motions of the plane $S E(2):=\left\{(x, y, \theta) \mid(x, y) \in \mathbb{R}^{2}, \theta \in \mathbb{R} / 2 \pi\right\}$

$$
(\dot{x}, \dot{y}, \dot{\theta})=u_{1}(\cos (\theta), \sin (\theta), 0)+u_{2}(0,0,1),
$$

where $x, y$ are coordinates on the plane and $\theta$ represents the angle of rotation of the car. Since we forbid backwards displacements, we impose $u_{1} \geq 0$. We want to minimize

$$
\mathscr{C}[q(.)]=\int_{0}^{T} \sqrt{u_{1}^{2}+u_{2}^{2}},
$$

with the following boundary conditions:

$$
q(0)=\left(x_{0}, y_{0}, \theta_{0}\right), \quad q(T)=\left(x_{1}, y_{1}, \theta_{1}\right) .
$$

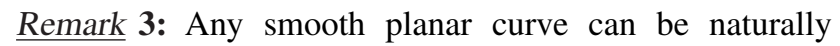
transformed into an admissible trajectory of this control system. Indeed, given $\gamma(t)=(x(t), y(t))$, we define

$$
q(t):=(x(t), y(t), \theta(t)),
$$

where $\theta(t)$ is the angle of the tangent vector with respect to the positive $x$-semiaxis. This is called the lift of $\gamma$. We then find suitable controls $u_{i}$ corresponding to $q(t)$ defined above. In this framework $u_{1}$ plays the role of $\|\dot{\gamma}\|$, while $u_{2}$ is $\|\dot{\gamma}\| K_{\gamma}$. Hence, the cost (5) coincides with $J[\gamma]$ defined in (2). Moreover, boundary conditions with orientation can be easily translated to boundary conditions on $S E(2)$.

Notice that, on the contrary, not all trajectories of (4) are lifts of planar curves, for example a trajectory with $u_{1} \equiv 0$.

We will prove that for the optimal control problem (4)-(5) on $S E(2)$ we have existence of minimizers. Starting from a minimizer of this problem, we will find counterexamples to the existence of minimizers of $J$ on $\mathscr{D}_{1}$ and $\mathscr{D}_{2}$.

For problems with projective boundary conditions, we study the dynamics given by (4) where we admit also backwards displacements (it is the Reeds-Shepp car, see [19]). In this case we don't have to impose $u_{1} \geq 0$ and we identify $(x, y, \theta) \simeq(x, y, \theta+\pi)$. Hence this dynamics is naturally defined on the quotient space $P T \mathbb{R}^{2}:=S E(2) / \simeq$. We choose the same cost (5). Also in this case, it is possible to lift planar curves to curves on $P T \mathbb{R}^{2}$. Projective boundary conditions can be easily translated to conditions on $(x, y, \theta) \in P T \mathbb{R}^{2}$.

For the optimal control problem (4)-(5) on $P T \mathbb{R}^{2}$ we have existence of minimizers with $L^{1}$ optimal controls. Its consequence on the problem of planar curves is the existence of minimizers of $J$ in $\mathscr{D}_{3}$. 
For both optimal control problems, the basic tool we use to compute minimizers is the Pontryagin Maximum Principle (PMP in the following), see [18]. It gives a necessary first-order condition for minimizers. Solutions of PMP are called extremals, hence minimizers have to be found among extremals. For details, see e.g. [1].

The structure of the paper is the following. In Section II we introduce the group $S E(2)$ and the space $P T \mathbb{R}^{2}$, and we define the optimal control problems on these spaces corresponding to the ones defined in Section I on the plane. We then study the optimal control problems and find some properties of minimizers.

Section III contains the main results of the paper: we prove Propositions 1-2-3 using properties of minimizers of problems studied in Section II.

\section{SOLUTION OF OPTIMAL CONTROL PROBLEMS}

In this section we recall the definition of the two optimal control problems given above. In the first we consider the Dubins' car [10]: it can both move forwards and rotate on itself. In the second we have the Reeds-Shepp car [19], that can move forwards, backwards and rotate on itself. Nevertheless, the problems we study are different than the ones studied in [10], [19]. We don't have constraints on velocity and curvature. We want instead to minimize (in both cases) a cost depending both on velocity and curvature.

\section{A. Dubins' car with length-curvature cost}

The Dubins' car is a car that can move both forwards and rotate on itself. The dynamics of the car is given by the following control system:

$$
(\dot{x}, \dot{y}, \dot{\theta})=u_{1}(\cos (\theta), \sin (\theta), 0)+u_{2}(0,0,1), \mathbf{u}_{\mathbf{1}} \geq \mathbf{0}
$$

with $u_{1}, u_{2} \in L^{1}([0, T], \mathbb{R})$. We impose $u_{1} \geq 0$ to forbid backwards displacements. Observe that $u_{1}$ is the planar velocity of the car and $u_{2}$ is its angular velocity. The controllability of this system can be checked by hand.

We fix a starting point $q_{0}=\left(x_{0}, y_{0}, \theta_{0}\right)$ and an ending point $q_{1}=\left(x_{1}, y_{1}, \theta_{1}\right)$. We want to minimize the cost

$$
\mathscr{C}[q(.)]=\int_{0}^{T} \sqrt{u_{1}^{2}+u_{2}^{2}}
$$

over all trajectories of (6) steering $q_{0}$ to $q_{1}$. Here the end time $T$ is fixed. After a suitable rototranslation, it is always possible to fix $q_{0}=(0,0,0)$. For this reason, from now on we will study only problems starting from $\mathrm{Id}=(0,0,0)$.

We now transform our optimal control problem in a minimum time problem, in which the dynamics is given again by (6), the cost is the time (that is free) and the constraint on the controls are $u_{1} \geq 0, u_{1}^{2}+u_{2}^{2} \leq 1$.
We apply Filippov existence theorem for minimum time problems, see e.g. [1, Cor 10.2], that gives a minimizer, hence $L^{1}$ optimal controls. It is a standard fact to restrict to $L^{\infty}$ optimal controls. For details see e.g. [22].

1) Computation of extremals: We now apply the PMP to the problem (6)-(7) transformed into a minimum time problem. For the expression of PMP for minimum time problems see e.g. [1, Ch. 12]. The control-dependent Hamiltonian of the system is

$$
H(q, \lambda, u)=\langle\lambda, \dot{q}\rangle=u_{1} h_{1}+u_{2} h_{2}
$$

where $h_{1}=\lambda_{x} \cos (\theta)+\lambda_{y} \sin (\theta), h_{2}=\lambda_{\theta}$, and $\lambda_{x}, \lambda_{y}, \lambda_{\theta}$ are the components of the covector $\lambda$ in the dual basis with respect to coordinates $(x, y, \theta)$.

We don't give a complete synthesis of the problem, since we only need to find a particular minimizer to use in proofs of Propositions 1-2. In particular, we don't consider abnormal extremals, for which we have $H=0$.

We consider instead normal extremals, for which we can choose $H=1$. Let us denote $\alpha$ and $\rho$ an angle and a positive number in such a way that $\lambda_{x}=\rho \cos (\alpha)$ and $\lambda_{y}=\rho \sin (\alpha)$.

Assume that at $t=t_{0}$ we have $h_{1}\left(t_{0}\right)>0$. Then PMP gives controls $u_{1}=h_{1}, u_{2}=h_{2}$, after having normalized $\left\|\left(h_{1}, h_{2}\right)\right\|=1$. Dynamics is given by

$$
\left\{\begin{array}{l}
\dot{x}=h_{1} \cos (\theta), \dot{y}=h_{1} \sin (\theta), \dot{\theta}=h_{2} \\
\dot{\lambda_{x}}=\dot{\lambda_{y}}=0, \dot{\lambda_{\theta}}=h_{1}\left(-\lambda_{x} \sin (\theta)+\lambda_{y} \cos (\theta)\right)
\end{array}\right.
$$

We have $\left|\theta\left(t_{0}\right)-\alpha\right|<\frac{\pi}{2}$ in $\mathbb{R} / 2 \pi$ and the equation on $\theta$ is

$$
2 \ddot{\theta}=\rho^{2} \sin (2(\theta-\alpha)) \text {. }
$$

It is the equation of the pendulum with $\theta=\alpha$ being the unstable equilibrium. This implies that when starting with $\left|\theta\left(t_{0}\right)-\alpha\right|<\frac{\pi}{2}$ in $\mathbb{R} / 2 \pi$, then $\theta$ will reach a value such that $\left|\theta\left(t_{0}\right)-\alpha\right|>\frac{\pi}{2}$ in $\mathbb{R} / 2 \pi$. Hence the corresponding extremal will have a time $t_{1}>t_{0}$ for which $h_{1}\left(t_{1}\right)<0$.

Assume now that at $t=t_{0}$ we have $h_{1}\left(t_{0}\right) \leq 0$. PMP gives controls $u_{1}=0$ and $u_{2}=\operatorname{sign}\left(h_{2}\right)$ and the extremal is a rotation on itself. Indeed, the dynamics is given by

$$
\left\{\begin{array}{l}
\dot{x}=\dot{y}=\dot{\lambda_{x}}=\dot{\lambda_{y}}=\dot{\lambda_{\theta}}=0 \\
\dot{\theta}=\operatorname{sign}\left(h_{2}\right) .
\end{array}\right.
$$

Since $\lambda_{x}$ and $\lambda_{y}$ are constant, either $\lambda_{x}=\lambda_{y}=0$ all along the extremal (thus $h_{1} \equiv 0$ ) or at least one of them is nonvanishing (thus there exists $t_{1}>t_{0}$ such that $h_{1}\left(t_{1}\right)>0$ ).

As already stated, for an extremal satisfying $h_{1}\left(t_{0}\right)>0$ (resp $h_{1}\left(t_{0}\right)<0$ ) there exists a time $t_{1}>t_{0}$ such that $h_{1}\left(t_{1}\right)<0$ (resp $\left.h_{1}\left(t_{1}\right)>0\right)$. Thus an extremal is the concatenation of trajectories satisfying (10) and trajectories satisfying (9). Consider an arc $\gamma\left(\left[t_{0}, t_{1}\right]\right)$ satisfying (10) between two arcs satisfying (9). Then the variation of $\theta$ along this arc should be $\pi$ because $\theta\left(t_{0}\right)=\alpha+\frac{\pi}{2} \bmod \pi$ 
and we should come back to $\theta\left(t_{1}\right)=\alpha+\frac{\pi}{2} \bmod \pi$ at the end with the dynamics $\dot{\theta}=1$. Moreover, one can prove that a concatenation of dynamics (9), (10) and (9) is never optimal.

Remark 4: A consequence of this study to the planar problem of minimization of $J$ on $\mathscr{D}_{2}$ is that planar curves with cusps are extremal, but never minimizers.

2) An example of a minimizer: In this section we give an example of a minimizer $\mathbf{q}($.$) defined on a small interval$ $[0,2 \xi]$ and satisfying $(10)$ on $[0, \xi]$ and (9) on $[\xi, 2 \xi]$. This trajectory is the basic example that we will use to prove non-existence of minimizers of cost (2) both on $\mathscr{D}_{1}$ and $\mathscr{D}_{2}$, i.e. Propositions 1 and 2.

Consider a trajectory $q^{1}(t)$ starting from Id, with given $\lambda_{x}=-\frac{1}{\sqrt{2}}, \lambda_{y}=0, \lambda_{\theta}=\frac{1}{\sqrt{2}}$. All quantities related to this trajectory are denoted with superscript 1 . Since $h_{1}(0)<0$, dynamics is given by (10) on an interval $\left[0, t^{1}\right]$ and we have

$$
\begin{array}{ll}
x^{1}\left(t^{1}\right)=y^{1}\left(t^{1}\right)=\lambda_{y}^{1}\left(t^{1}\right)=0, & \lambda_{x}^{1}\left(t^{1}\right)=-\frac{1}{\sqrt{2}}, \\
\theta^{1}\left(t^{1}\right)=t^{1}, & \lambda_{\theta}^{1}\left(t^{1}\right)=\frac{1}{\sqrt{2}} .
\end{array}
$$

We choose $t^{1}=\frac{\pi}{2}$ and observe that $h_{1}^{1}\left(t^{1}\right)=0$. Recall that on this interval controls are $u_{1}^{1}=0, u_{2}^{1}=1$.

Then dynamics is given by (9) on an interval $\left[t^{1}, t^{1}+s^{1}\right]$. Since $\lambda_{\theta}^{1}$ is continuous, so $u_{2}^{1}$ is. Then $\theta^{1}(t)=\frac{\pi}{2}+\left(t-t^{1}\right)+$ $o\left(t-t^{1}\right)$ on $\left(t^{1}, t^{1}+s^{1}\right)$, thus $h_{1}^{1}(t)>0$ on $\left(t^{1}, t^{1}+s^{1}\right)$ for a sufficiently small choice of $s^{1}$. As a consequence, $x(t) \neq 0$, $y(t) \neq 0$ for all $t \in\left(t^{1}, t^{1}+s^{1}\right)$. Recall now that all normal extremals are local minimizers, see e.g. [1, Cor 17.1]. We apply this result to $q^{1}(t)$ in $t^{1}$ and find a $\varepsilon^{1}$ such that $q^{1}(t)$ is a minimizer over $\left[t^{1}-\varepsilon^{1}, t^{1}+\varepsilon^{1}\right]$.

We now prove that for a small $\xi<\varepsilon^{1}$ the trajectory $q^{1}(t)$ is not only a minimizer, but the unique minimizer steering $Q_{0}=q^{1}\left(t^{1}-\xi\right)$ to $Q_{1}=q\left(t^{1}+\xi\right)$. We prove it by contradiction. Assume that there exists another minimizer $q^{2}$ steering $Q_{0}$ to $Q_{1}$. In the following all quantities related to this minimizer are denoted with superscript 2 . As a consequence of the existence of $q^{2}$, we have another minimizer $q^{3}($.$) steering q^{1}\left(t^{1}-\varepsilon^{1}\right)$ to $q^{1}\left(t^{1}+\varepsilon^{1}\right)$, given by the concatenation of $q^{1}$ on $\left[t^{1}-\varepsilon^{1}, t^{1}-\xi\right]$, then $q^{2}$ on $\left[t^{1}-\xi, t^{1}+\xi\right]$, then again $q^{1}$ on $\left[t^{1}+\xi, t^{1}+\varepsilon^{1}\right]$. See Figure 3. Since $q^{3}$ is a minimizer, then it is a solution of PMP. As a consequence, its tangent covector is continuous. For this reason, we have $\lambda^{1}\left(t^{1}-\xi\right)=\lambda^{2}\left(t^{1}-\xi\right)$. Since this covector satisfies $h_{1}<0$, then trajectory $q^{3}$ satisfies dynamics given by (10) on a neighborhood of $t^{1}-\xi$, hence $q^{1}$ and $q^{2}$ coincide on this neighborhood due to uniqueness of solution for (10). We can prove in the same way that $q^{1}$ and $q^{2}$ coincide on the whole interval $\left[t^{1}-\xi, t^{1}\right)$. Similarly, we have $\lambda^{1}\left(t^{1}+\xi\right)=\lambda^{2}\left(t^{1}+\xi\right)$, hence $q^{1}$ and $q^{2}$ coincide in the whole interval $\left(t^{1}, t^{1}+\xi\right]$ due to uniqueness of solution for (9). Finally, they coincide also in $t^{1}$ due to continuity. Hence $q^{2}=q^{1}$ on the interval $\left[t^{1}-\xi, t^{1}+\xi\right]$. Contradiction.

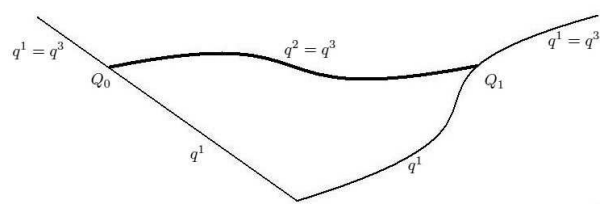

Fig. 3. Construction of trajectory $q^{3}$.

We now define the trajectory $\mathbf{q}$ in $S E(2)$, using $q^{1}$ defined on the interval $\left[t^{1}-\xi, t^{1}+\xi\right]$. We first perform a left multiplication of $q^{1}$ in order to have $q^{1}\left(t^{1}\right)=\mathrm{Id}$, then a time shift $\left[t^{1}-\xi, t^{1}+\xi\right] \mapsto[0,2 \xi]$. The resulting trajectory is $\mathbf{q}(t):=\left(q^{1}\left(t^{1}\right)\right)^{-1} q^{1}\left(t+t^{1}-\xi\right)$. We thus have that $\mathbf{q}$ is the unique minimizer steering $\mathbf{q}(0)$ to $\mathbf{q}(2 \xi)$, and it satisfies dynamics (10) on $[0, \xi]$ and (9) on $[\xi, 2 \xi]$.

\section{B. Projective Reeds-Shepp car with length-curvature cost}

The Reeds-Shepp car is a car that can move forwards, backwards and rotate on itself. The set of configurations can be identified with a quotient of the group of motions of the plane $P T \mathbb{R}^{2}:=S E(2) / \simeq$ where $(x, y, \theta) \simeq(x, y, \theta+\pi)$. For a better comprehension we use the same notation used for $S E(2)$, omitting the identification. We also omit checks of good definitions of dynamics and cost given below.

The dynamics is given by the following control system:

$$
(\dot{x}, \dot{y}, \dot{\theta})=u_{1}(\cos (\theta), \sin (\theta), 0)+u_{2}(0,0,1),
$$

with $u_{1}, u_{2} \in L^{1}([0, T], \mathbb{R})$. Fix starting and ending points $q_{0}=\left(x_{0}, y_{0}, \theta_{0}\right), q_{1}=\left(x_{1}, y_{1}, \theta_{1}\right)$. We want to minimize

$$
\mathscr{C}[q(.)]=\int_{0}^{T} \sqrt{u_{1}^{2}+u_{2}^{2}}
$$

over all trajectories of (11) steering $q_{0}$ to $q_{1}$. Here the end time $T$ is fixed. Also in this case, due to invariance by rototranslation of both the dynamics and the cost, we specialize to problems starting from $\operatorname{Id}=(0,0,0)=(0,0, \pi)$.

Controllability is a direct consequence of Rashevsky-Chow theorem (see e.g. [1]) for this problem.

1) Computation of extremals: In this section we compute minimizers for our optimal control problem. We follow procedure presented in Sections II-A.

First transform the optimal control problem (11)-(12) in a minimal time problem where dynamics is given again by (11) and controls are bounded by $u_{1}^{2}+u_{2}^{2} \leq 1$. We then prove that this problem admits a minimum for all pairs of starting and ending points and we restrict ourselves to $L^{\infty}$ optimal controls. We then apply PMP, using its expression for minimal time problem. Since dynamics (11) on $P T \mathbb{R}^{2}$ 
coincides locally with dynamics (6) on $S E(2)$, we have the same control-depending Hamiltonian

$$
H(q, \lambda, u)=\langle\lambda, \dot{q}\rangle=u_{1} h_{1}+u_{2} h_{2}
$$

where $h_{1}=\lambda_{x} \cos (\theta)+\lambda_{y} \sin (\theta), h_{2}=\lambda_{\theta}$, and $\lambda_{x}, \lambda_{y}, \lambda_{\theta}$ are the components of the covector $\lambda$ in the dual basis with respect to coordinates $(x, y, \theta)$. We can neglect abnormal extremals, since they are trajectories reduced to a point.

We fix $H=1$ and observe that we don't have condition $u_{1} \geq 0$ in this case. Hence solutions of the PMP are given by $u_{1}=h_{1}, u_{2}=h_{2}$. We thus have

$$
\left\{\begin{array}{l}
\dot{x}=h_{1} \cos (\theta), \dot{y}=h_{1} \sin (\theta), \dot{\theta}=h_{2}, \\
\dot{\lambda_{x}}=\dot{\lambda_{y}}=0, \dot{\lambda_{\theta}}=h_{1}\left(-\lambda_{x} \sin (\theta)+\lambda_{y} \cos (\theta)\right) .
\end{array}\right.
$$

The explicit solution of this problem is given in [14], [20], [21] in the case of $S E(2)$. For our treatment, it is sufficient to observe some properties of extremals. First of all, they are completely determined by the initial covector $\lambda$, due to uniqueness of solution of (14). Moreover, the solution is analytic. Hence we have only one of these possibilities:

- either $h_{1} \equiv 0$, and the corresponding extremals are $q(t)=\left(0,0, \theta^{a}(t)\right)$

- or $h_{1}$ has only a finite number of times in which it is vanishing, hence the corresponding trajectory $q($.$) has$ only a finite number of points in which $\dot{x}=\dot{y}=0$.

Notice that trajectories of the second kind can be "well projected" to the plane, i.e. it holds

Lemma 1: Let $q(t)=(x(t), y(t), \theta(t))$ be an extremal for the optimal control problem (11)-(12) for which $h_{1}$ is vanishing only for a finite number of times $t_{1}, \ldots, t_{n}$. Let $p(t)=\Pi(q(t))$ be the projection of $q$ on the plane via $\Pi:(x, y, \theta) \mapsto(x, y)$. Then for each time $t \in[0, T]$ we have either $\dot{p}(t) \approx(\cos (\theta(t)), \sin (\theta(t)))$ or $\dot{p}\left(t_{0}\right)=0$ and $\lim _{\tau \rightarrow t^{-}} \frac{\dot{p}(\tau)}{\|\dot{p}(\tau)\|} \approx \lim _{\tau \rightarrow t^{+}} \frac{\dot{p}(\tau)}{\|\dot{p}(\tau)\|} \approx(\cos (\theta(t)), \sin (\theta(t)))$.

Proof: Notice that $\dot{p}=\left(u_{1} \cos (\theta), u_{1} \sin (\theta)\right)$ since $q$ satisfies (11). Hence $\dot{p}(t) \approx(\cos (\theta(t)), \sin (\theta(t)))$ if $u_{1}(t) \neq$ 0 . If instead $u_{1}(t)=0$, then there exists $(t-\varepsilon, t+\varepsilon)$ on which $u_{1}(\tau) \neq 0$ for all $\tau \neq t$. Thus $\frac{\dot{p}(\tau)}{\|\dot{p}(\tau)\|}=$ $\frac{\left(u_{1}(\tau) \cos (\theta(\tau)), u_{1}(\tau) \sin (\theta(\tau))\right)}{\left|u_{1}(\tau)\right|} \approx(\cos (\theta(\tau)), \sin (\theta(\tau)))$. Passage to limit gives the result in $t$.

Remark 5: An interesting property (see [21]) of this second family of extremals is that there are minimizers with one or two points in which $u_{1}=0$, but trajectory with three or more points in which $u_{1}=0$ are never minimizers. Thus minimizers for $J$ over the set $\mathscr{D}_{3}$ may present one or two cusps, but not more than two.

We will use in the following these properties to prove existence of a minimizer of $J$ over all curves in $\mathscr{D}_{3}$. Notice that Lemma 1 doesn't hold for minimizers of the problem on $S E(2)$ defined in Section II-A.

\section{SOlution OF PROBLEMS AND EXISTENCE OF MINIMIZERS}

This section contains the main results of the paper. We first prove Propositions 1 and 2, i.e. the non-existence of minimizers of $J$ in $\mathscr{D}_{1}$ and $\mathscr{D}_{2}$. On the contrary, we prove Proposition 3 , i.e. the existence of minimizer of $J$ in $\mathscr{D}_{3}$.

\section{A. Boundary conditions with orientation: non-existence of} minimizers

In this section we give a counterexample to the existence of minimizers of $J$ for boundary conditions with orientation. We prove it both in the case in which curves are chosen to be in $\mathscr{D}_{1}$ and in $\mathscr{D}_{2}$. The basic idea is to lift the planar problem to the problem on $S E(2)$, then solve the problem on $S E(2)$ and finally project it on the plane. But this last step doesn't work well, since in the case we present below the projection of the solution of the problem on $S E(2)$ doesn't satisfy boundary conditions with orientation fixed at the beginning.

Start considering the trajectory $\mathbf{q}(t)=(\mathbf{x}(t), \mathbf{y}(t), \theta(t))$ on $S E(2)$ defined in Section II-A.2. Define its projection $\mathbf{p}(t):=\Pi(\mathbf{q}(t))$ via the map $\Pi:(x, y, \theta) \mapsto(x, y)$. As already stated, $\dot{\mathbf{p}}=0$ on $(0, \xi)$ and $\dot{\mathbf{p}} \neq 0$ on $(\xi, 2 \xi)$.

We then define a sequence of planar curves $p^{n}$ on $(0,2 \xi)$, each of them satisfying the following boundary conditions with orientation: $p^{n}(0)=\mathbf{p}(0), p^{n}(2 \xi)=\mathbf{p}(2 \xi), \dot{p}^{n}(0) \sim$ $(\cos (\theta(0)), \sin (\theta(0))), \dot{p}^{n}(2 \xi) \sim \dot{\mathbf{p}}(2 \xi)$. We also require that $p^{n}$ converges to $\mathbf{p}$, while $J\left[p^{n}\right]$ converges to $\mathscr{C}[\mathbf{q}]$. We define the curve $p^{n}$ with a geometric construction, see Figure 4. First define $p^{n}$ on $\left[\xi+\frac{\xi}{n}, 2 \xi\right]$ coinciding with $\mathbf{p}$. Then define the point $C:=\mathbf{p}\left(\xi+\frac{\xi}{n}\right)$ and draw the line $r$ that is the tangent to $\mathbf{p}$ at $C$. Then draw the line $s$ connecting $O=(0,0)$ and $(\cos (\theta(0)), \sin (\theta(0)))$. Since $r$ and $s$ are not parallel, they have an intersection point $B$.

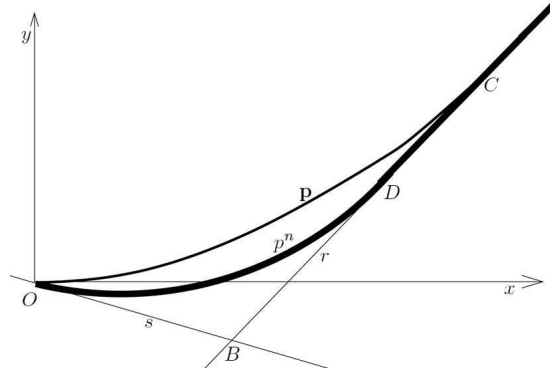

Fig. 4. Construction of the trajectory $p^{n}$ (case $\mathscr{L}(O B) \leq \mathscr{L}(B C)$ ).

Then we have two cases:

- If $\mathscr{L}(O B) \leq \mathscr{L}(B C)$, fix point $D$ on $B C$ such that $\mathscr{L}(O B)=\mathscr{L}(B D)$ and define the arc $\overparen{O D}$ that is tangent to $O B$ in $O$ and to $B C$ in $D$. Define $p^{n}$ on $\left(0, \xi+\frac{\xi}{n}\right)$ as the concatenation of $\overparen{O D}$ and $\overline{D C}$.

- If $\mathscr{L}(O B) \geq \mathscr{L}(B C)$, fix $D$ on $O B$ satisfying $\mathscr{L}(B D)=\mathscr{L}(B C)$ and make the construction of the 
arc $\overparen{D C}$. Define $p^{n}$ on $\left(0, \xi+\frac{\xi}{n}\right)$ as the concatenation of $\overline{O D}$ and $\overparen{D C}$.

Notice that all $p^{n}$ satisfy boundary conditions with orientations and that the sequence converges to p. Moreover, $J\left[p^{n}\right]$ restricted to the interval $\left[\xi+\frac{\xi}{n}, 2 \xi\right]$ coincides with $J[\mathbf{p}]$ on the same interval, that in turns coincides with $\mathscr{C}[\mathbf{q}]$ on the same interval (see Remark 3). Concerning the interval $\left[0, \varepsilon+\frac{\varepsilon}{n}\right]$, we have $C \rightarrow B \rightarrow O$ for $n \rightarrow \infty$, hence $J[D C]$ or $J[O D]$ tends to 0 . Instead, $J[\overparen{O D}]$ or $J[\overparen{D C}]$ tends to $-\theta(0)$. Indeed, assume that $\mathscr{L}(O B) \leq \mathscr{L}(B C)$ and compute $J[\overparen{O D}]$ parametrized by arclength. Here $\int_{a}^{b} K_{\gamma}=$ $\alpha_{\gamma}(b)-\alpha_{\gamma}(a)$ where $\alpha_{\gamma}(t)$ is the angle of $\dot{\gamma}(t)$, thus

$$
\begin{array}{r}
\alpha_{\overparen{O D}}(D)-\alpha_{\overparen{O D}}(O)=\int_{0}^{\mathscr{L}(\overparen{O D})} K_{\gamma} \leq J[\overparen{O D}] \leq \\
\leq \int_{0}^{\mathscr{L}(\overparen{O D})}\left(1+K_{\gamma}\right)=\mathscr{L}(\overparen{O D})+\alpha_{\overparen{O D}}(D)-\alpha_{\overparen{O D}}(O) .
\end{array}
$$

The result follows recalling that $\alpha_{\overparen{O D}}(D) \rightarrow \theta\left(\xi+\frac{\xi}{n}\right) \rightarrow$ $\theta(\xi)=0$. The case $\mathscr{L}(O B) \geq \mathscr{L}(B C)$ is similar.

We have thus defined a sequence of curves $p^{n} \in \mathscr{D}_{1} \subset \mathscr{D}_{2}$ minimizing the cost $J$ but such that the limit curve $\mathbf{p}$ does not satisfy boundary conditions with orientation, since $\dot{\mathbf{p}}=0$ on $(0, \xi)$ and $\dot{\mathbf{p}}\left(\xi^{+}\right) \sim(1,0)$. Hence $\mathbf{p} \notin \mathscr{D}_{2}$. We prove that it implies the non-existence of a minimizer for these boundary conditions. By contradiction, assume that a minimizer of $J$ exists in $\mathscr{D}_{2}$. Thus its lift $\bar{q}$ to $S E(2)$ is a minimizer for $\mathscr{C}$ between $\mathbf{q}(0)$ and $\mathbf{q}(2 \varepsilon)$, that is impossible since $\mathbf{q}$ is the unique minimizer between the two points. Contradiction.

\section{B. Projective boundary conditions: existence of minimizers}

In this section we prove existence of a minimizing curve in $\mathscr{D}_{3}$ for all choices of projective boundary conditions, i.e. we prove Proposition 3. The basic idea is that also in this case we can lift the problem of planar curves to the problem on $P T \mathbb{R}^{2}$ defined above, solve it and then project the solution to the plane. But in this case the whole procedure works well.

Start fixing projective boundary conditions, i.e. fix a starting point $\left(x_{0}, y_{0}\right)$ with direction $v_{0}$ and an ending point $\left(x_{1}, y_{1}\right)$ with direction $v_{1}$. Assume that $\left(x_{0}, y_{0}\right) \neq\left(x_{1}, y_{1}\right)$ and $v_{0}, v_{1}$ are nonvanishing vectors. Recall that we want to find a curve $\gamma \in \mathscr{D}_{2}$ such that $\gamma(0)=\left(x_{0}, y_{0}\right), \dot{\gamma}(0) \approx v_{0}$, $\gamma(T)=\left(x_{1}, y_{1}\right), \dot{\gamma}(T) \approx v_{1}$ and that is a minimizer of $J$.

Consider the optimal control defined on $P T \mathbb{R}^{2}$ presented in Section II-B with the following starting and ending point: $q_{0}=\left(x_{0}, y_{0}, \theta_{0}\right)$ and $q_{1}=\left(x_{1}, y_{1}, \theta_{1}\right)$ where each $\theta_{i}$ is the angle formed by the vector $v_{i}$ with respect to the $x$ axis. Then solve the problem and call $\mathbf{q}($.$) the minimizing$ trajectory (that is not necessary unique). The basic remark is that $\mathbf{q}$ is of the second kind (see Section II-B.1), since $\left(x_{0}, y_{0}\right) \neq\left(x_{1}, y_{1}\right)$. As proved in Lemma 1 , in this case $\dot{\mathbf{p}} \approx$ $(\cos (\theta), \sin (\theta))$ except for a discrete set of points $t_{1}, \ldots, t_{n}$ on which we have the weaker property $\lim _{\tau \rightarrow t_{i}} \frac{\dot{\mathbf{p}}}{\|\dot{\mathbf{p}}\|} \approx$ $(\cos (\theta), \sin (\theta))$. If we have at the starting point $\dot{\mathbf{p}}(0) \neq$ 0 , then $\mathbf{p}$ satisfies projective boundary conditions at the beginning. Otherwise reparametrize $\mathbf{p}$ by arclength in an interval $[0, \varepsilon]$, and find that $\mathbf{p}$ satisfies boundary conditions at the beginning. The same result can be proved for the ending point. Hence $\mathbf{p}$ satisfies projective boundary conditions.

We now prove that $\mathbf{p}$ is a minimizer of $J$, by contradiction. Assume that there exists $\bar{p}$ satisfying the same projective boundary conditions and such that $J[\bar{p}]<J[\mathbf{p}]$. Thus its lift $\bar{q}$ steers $q_{0}$ to $q_{1}$ and satisfies $\mathscr{C}[\bar{q}]<\mathscr{C}[\mathbf{q}]$, hence $\mathbf{q}$ is not a minimizer. Contradiction.

\section{REFERENCES}

[1] A.A. Agrachev, Yu. L. Sachkov, Control Theory from the Geometric Viewpoint, Encyclopedia of Math. Sciences, v. 87, Springer, 2004.

[2] G. Bellettini, Variational approximation of functionals with curvatures and related properties, J. Convex Anal., v. 4, no. 1, 91-108, 1997.

[3] A. Bloch, Nonholonomic Mechanics and Control, Interdisciplinary Applied Mathematics, Vol. 24, Springer, 2003.

[4] U. Boscain, F. Rossi, Invariant Carnot-Caratheodory metrics on $S^{3}$ $S O(3), S L(2)$ and lens spaces, SIAM J. Control Optim. 47, no. 4, pp. 1851-1878, 2008.

[5] U. Boscain, F. Rossi, Projective Reeds-Shepp car on $S^{2}$ with quadratic cost, to appear in Control, Optimisation and Calculus of Variations.

[6] R. Brockett, Control theory and singular Riemannian geometry, in New directions in applied mathematics, (Cleveland, Ohio, 1980), Springer, New York-Berlin, pp. 11-27, 1982.

[7] F. Cao, Y. Gousseau, S. Masnou, P. Pérez, Geometrically guided exemplar-based inpainting, to appear.

[8] G. Citti, A. Sarti, A cortical based model of perceptual completion in the roto-translation space, J. Mat. Im. Vis. 24, n. 3, p. 307-326, 2006

[9] I. Coope, Curve interpolation with nonlinear spiral splines, IMA J. Num. An. 13, no. 3, pp. 327-341, 1993

[10] L. E. Dubins, On curves of minimal length with a constraint on average curvature, and with prescribed initial and terminal positions and tangents, Amer. J. Math., vol. 79, no. 3, pp. 497-516, 1957.

[11] V. Jurdjevic, Geometric Control Theory, Cambridge Univ, Press, 1997

[12] A. Linnér, Existence of free nonclosed Euler-Bernoulli elastica, Nonlin. Anal. 21, no. 8, pp. 575-593, 1993.

[13] A. Linnér, Curve-straightening and the Palais-Smale condition, Trans. AMS 350 (9), pp. 3743-3765, 1998.

[14] I. Moiseev, Yu. L. Sachkov, Maxwell strata in sub-Riemannian problem on the group of motions of a plane, to appear, arXiv: 0807.4731.

[15] R. Montgomery, A Tour of Subriemannian Geometries, their Geodesics and Applications, Math. Surveys and Monographs 91, AMS, 2002.

[16] J. Petitot, Vers une Neuro-géomètrie. Fibrations corticales, structures de contact et contours subjectifs modaux, Math. Inform. Sci. Humaines No. 145, pp. 5-101, 1999.

[17] J. Petitot, Neurogéomètrie de la vision - Modèles mathématiques et physiques des architectures fonctionnelles, Les Éditions de l'École Polythecnique, 2008.

[18] L.S. Pontryagin et al., The Mathematical Theory of Optimal Processes, John Wiley and Sons, Inc., 1961.

[19] J. A. Reeds, L. A. Shepp, Optimal paths for a car that goes both forwards and backwards, Pacific J. Math., v. 145, n. 2, 367-393, 1990.

[20] Yu. L. Sachkov, Conjugate and cut time in sub-Riemannian problem on the group of motions of a plane, ESAIM: COCV, to appear.

[21] Yu. L. Sachkov, Cut locus and optimal synthesis in the sub-Riemannian problem on the group of motions of a plane, submitted.

[22] A.V. Sarychev, D. F. M. Torres, Lipschitzian regularity of minimizers for optimal control problems with control-affine dynamics, Appl. Math. Optim. 41, no. 2, pp. 237-254, 2000. 